\title{
LEER Y ESCRIBIR HISTÓRICAMENTE: LOS DESAFÍOS PENDIENTES DE LA ENSEÑANZA Y DEL APRENDIZAJE DE LA HISTORIA
}

\author{
READING AND WRITING HISTORICALLY: UNRESOLVED CHALLENGES \\ IN TEACHING AND LEARNING OF HISTORY
}

\author{
Rodrigo Henríquez Vásquez* y Yerko Muñoz Salinas**
}

\begin{abstract}
El propósito de este artículo es ofrecer una estado de la cuestión acerca de uno de los tópicos más relevantes en educación histórica del último tiempo: la alfabetización histórica y las formas comunicativas en donde se expresa el aprendizaje histórico. Se caracterizan algunas definiciones conceptuales y líneas de investigación que sostienen que el desarrollo del lenguaje académico es el principal desafío de la educación histórica en la actualidad.
\end{abstract}

Palabras claves: Alfabetización histórica, desafíos educacionales, enseñanza en Chile.

The focus of this paper is review a brief state-of-art of one of the most relevant topics of historical education nowadays: historical literacy and the ways throughout the historical learning is expressed. Several concepts are provided and characterized alongside the main research streams which point out the importance of academical language in order to enhance the historical and educational challenges today.

Key words: Historical literacy, educational challenges, teaching in Chile.

\section{Introducción}

El objetivo principal del artículo que a continuación se presenta es la realización de un panorama concerniente a dos tópicos emergentes en la discusión contemporánea concerniente a alfabetización histórica, a saber: el desarrollo de la lectura y el de la escritura en estudiantes secundarios. A partir del ofrecimiento de un estado del arte del campo, se intentará proponer un diálogo entre algunos problemas del ámbito y los hallazgos más actuales de las líneas de investigación y trabajo empírico del entorno nacional e internacional.

Como punto de partida se sugiere identificar el desarrollo investigativo de lo que se ha denominado "razonamiento histórico" o "pensamiento histórico" como el principal foco de atención de los estudios dedicados a la enseñanza y al aprendizaje de la historia en la actualidad (Van Boxtel \& Van Drie, 2008; Wineburg, 2001). No obstante lo anterior, la tradición iberoamericana ha utilizado la expresión "didáctica de la historia" para referirse a las investigaciones que incluyen el estudio del "pensamiento histórico", aun cuando este no sea su foco principal. A diferencia de esta tradición, los estudios provenientes del mundo anglosajón han utilizado la expresión historical literacy (que en este artículo traducimos como "alfabetización histórica") para denominar un foco parecido al de la didáctica de la historia, pero focalizado en las formas y modalidades por medio de las que se adquiere el lenguaje disciplinar de la historia. Se considera, a partir de este enfoque, que es desde y mediante el lenguaje como se generan las oportunidades más eficaces para propiciar los aprendizajes, las que a su vez inciden también en las prácticas de enseñanza (Snow \& Uccelli, 2009). En este artículo se caracterizarán algunos ámbitos de estudio y desarrollo de la alfabetización histórica tanto en el dominio escrito como en el oral en prácticas de aula.

¿Qué es la alfabetización disciplinar? Resumidamente, se trata de un campo de estudio cuyo foco es el impulso de prácticas disciplinares en donde se desarrolle el acceso, producción y transformación del conocimiento (Moje, 2008). Estas prácticas se han focalizado en las habilidades

\footnotetext{
* Pontificia Universidad Católica de Chile, Facultad de Educación, Instituto de Historia. Santiago, Chile. Correo electrónico: rodrigo.henriquez@uc.cl

** Programa de Doctorado en Educación, Pontificia Universidad Católica de Chile. Santiago, Chile. Correo electrónico: ymunozsalinas@gmail.com
} 
de lectura y escritura propias de las disciplinas científicas y sociales, además del modo en que estas habilidades pueden desplegarse en la educación escolar secundaria (Moje, 2008; Shanahan \& Shanahan, 2008). Un ejemplo de lo anterior radica en el uso de conceptos propios de los diversos metalenguajes, como tesis, evidencia y análisis, los que adquieren rasgos particulares a raíz de su uso en cada campo disciplinar (Monte-Sano, 2010). Estas caracterizaciones han sido realizadas en el contexto escolar estadounidense, por lo que presentan muchas diferencias a la realidad sudamericana y chilena.

Una gradación de los diversos niveles que estructuran la adquisición de la alfabetización académica de los estudiantes ha sido caracterizada por Shanahan y Shanahan (2008). En ella se identifican tres fases de la alfabetización en la educación secundaria. En primer lugar, los autores distinguen la alfabetización básica, que incluye las habilidades de decodificación $\mathrm{y}$ reconocimiento de palabras de alta frecuencia que subyacen a las diversas tareas de escritura. En esta etapa, los estudiantes se aproximan a los niveles organizacionales o estructurales del texto, tales como la estructura de textos expositivos simples y la formulación básica de los conceptos históricos implicados en la pregunta que orienta la tarea. En segundo lugar, se establece la alfabetización intermedia, que contempla las habilidades vinculadas a las estrategias genéricas de comprensión lectora y a la facultad de decodificar los significados de palabras comunes, así como el conocimiento de formas más complejas de organización textual (tramas paralelas o circulares, problema-solución, causa-efecto) por medio de la identificación de la intención del autor como herramienta general para desarrollar respuestas críticas. En tercer lugar, es posible hallar la alfabetización disciplinar, que corresponde a las habilidades de lectura y escritura especializadas de Historia, Ciencias, Matemáticas, Literatura, u otras materias. Así, en esta fase, las habilidades precedentes de orden más general adquieren un carácter más específico y se transforman en características de un nivel más técnico y asociado a la naturaleza de las tareas disciplinarias.

La repercusión que se propone rescatar de lo anterior radica en que la alfabetización disciplinar requiere para su desarrollo el dominio del lenguaje académico de una disciplina. Snow y Ucelli (2008) definen lenguaje académico como un "dominio léxico gramatical, discursivo y disciplinario que permite "empaquetar" ideas complejas y abstractas al escribir, tanto como comprender los textos de la escuela y de las comunidades académicas" (Concha et al., 2010:91). Estudios relativos a lenguaje académico disciplinar de los profesores de Historia (Schleppegrell, Achugar \& Oteíza, 2004; Schleppegrel \& Oliveira, 2006) muestran cómo las formas lingüísticas se expresan de manera inseparable a las diversas disciplinas. Así, para llegar a dominar una de estas, los lectores y escritores deben construir sus argumentos y opiniones de manera discursiva en un texto, pero usando, necesariamente, los recursos del lenguaje académico (Coffin, 2006).

La estructura del texto se cimienta en dos ejes centrales: la alfabetización histórica y la lectoescritura en esta disciplina. Se exponen los conceptos fundamentales de cada uno de estos ejes y se revisan investigaciones empíricas que arrojan luz acerca de cómo poder propiciar mejores estrategias de evaluación de estas habilidades. Toda vez que se ha podido se han propuesto niveles, gradaciones y marcos orientadores de los hallazgos con el objeto de generar andamiajes para el análisis y puntos de referencias para las futuras investigaciones.

\section{Alfabetización histórica}

El término alfabetización histórica es una traducción del inglés historical literacy. Aunque también se ha utilizado la traducción "literacidad histórica", proponemos considerar el concepto de "alfabetización histórica" como el más preciso para aludir al aprendizaje de la historia vinculado a un lenguaje, además del modo en que este se habla y se escribe en el marco de una disciplina. Vale la pena recordar que uno de los fines educativos más extendidos en los siglos XIX y XX fue el de aprender a leer y a escribir para luego aprender las disciplinas escolares. El foco es el mismo en su base, pero apunta de manera más específica, es decir, a leer y escribir para aprender el lenguaje mismo de la disciplina.

La alfabetización histórica y disciplinar tiene como objetivo general que los y las estudiantes puedan aprender el lenguaje de una disciplina para utilizarlo en la comprensión y en la participación del mundo de la vida. Como se verá más adelante, muchos de los problemas de aprendizaje y de enseñanza son producidos por obstáculos vinculados al lenguaje de las disciplinas, en las que, en muchas veces, se señalan o se nombran de manera dificultosa algunas cosas que podrían resultar más simples. No obstante 
lo anterior, es justo recalcar que no es el objetivo de la alfabetización histórica formar historiadores e historiadoras como han señalado algunos de los críticos de esta perspectiva. El propósito de esta radica más bien en ofrecer a los estudiantes las oportunidades que la historiografía y su lenguaje entregan para tomar perspectiva del presente con el objeto de utilizarlo como un punto de vista orientado a la comprensión de la experiencia. Este propósito dota de una concreción práctica a la noción de "conciencia histórica" que atrajo los estudios de numerosos investigadores de la década de 1990 y 2000 (Rüsen 1992; Lee 2002) de las formas en las así llamadas "competencias narrativas de la conciencia histórica" influenciaban nuestra vida cotidiana. Sin embargo, un problema central para quienes trabajamos con esas categorías resultó ser la difícil adecuación de estas al contexto escolar. Las complejas teorías acerca de las competencias narrativas (de orientación, forma y función) que, por ejemplo, utilizó uno de los autores en su tesis de doctorado (Henríquez, 2008), resultaron mostrarse excesivamente abstractas a la hora de intentar llevarlas al aula con la intención de caracterizar, a modo de muestra, la capacidad de los estudiantes para poder establecer la continuidad y el cambio de un determinado problema histórico. Por ello, se consideró importante no solo caracterizar la dimensión heurística de la disciplina histórica, cuyo foco es la realización de preguntas, conjeturas y relaciones entre los textos de las fuentes y los conceptos o teorías que ayudan a contextualizarlas, sino que también la dimensión discursiva y, dentro de ella, la dimensión gramatical del lenguaje de la historia.

En el uso que proponemos, la noción de gramática no debe entenderse en un modo tradicionalmente, esto es, como un conjunto de normas a las que los significados están adheridos. Por el contrario, la gramática, como sostiene Michael Halliday (2004), uno de sus teóricos más influyentes del campo, estaría relacionada con la dimensión semántica del lenguaje. Conocer la gramática de la historia sería, por tanto, conocer los medios a partir de los cuales la disciplina histórica ha elaborado, desde sus propias formas de trabajo, su modo de construir su conocimiento y sus objetos. Por tanto, penetrar en esta dimensión implica entrar en el núcleo mismo de la enseñanza y el aprendizaje.

En consecuencia, el lenguaje puede convertirse para los neófitos del campo tanto en un obstáculo como en una oportunidad. Si el conocimiento histórico no puede comunicarse en textos, de diversa índole, o bien, en el discurso oral, dicho conocimiento no puede ser ni enseñado ni aprendido. Por ello, si la historia no puede ser expresada mediante textos elaborados por estudiantes o declarados en forma oral por estos, no se hace posible para quienes los evalúen asegurar la existencia de un aprendizaje profundo de la disciplina en los alumnos, así como tampoco cotejar la alineación entre su desempeño y objetivos de aprendizaje como "comprender" o "analizar".

En el contexto chileno, un ejemplo de lo anterior puede referirse con relación a un aprendizaje esperado del nivel de $3^{\circ}$ Medio. En este caso, el Aprendizaje Esperado 16 (AE16) prescribe: "[A] nalizar, apoyándose en diversas fuentes, los factores que inciden en el fin de la dictadura militar y los procesos que dan inicio a la transición a la democracia durante la década de 1980 (itinerario de la transición, formación de bloques políticos, plebiscito de 1988, negociación de las reformas constitucionales, elección presidencial de 1989)" (MINEDUC 2015:33). Un desglose de la indicación permite señalar que "analizar" factores en diversas fuentes exige habilidades de lectura e identificación de factores para luego permitir la integración de las posiciones de las evidencias y así elaborar un análisis. Según sostienen autores como Lee (2005) y Monte-Sano (2010), para que un estudiante pueda sortear exitosamente el AE16 deberá contar con un conjunto de habilidades de lectura e identificación de los argumentos para seleccionarlos y solo así, en una segunda instancia y dependiendo del propósito de escritura, elaborar un texto o una exposición oral, de ahí pues que el lenguaje disciplinar entrañe una ambivalencia de "obstáculo" y "oportunidad".

Concebir al lenguaje disciplinar como "transparente" o argumentar que el sector de Lenguaje y Comunicación debe encargarse de ello mientras nosotros, los profesores de Historia y Ciencias Sociales, nos dedicamos a enseñar "solo" historia, no solo es a nuestro juicio un error, sino que una secuela más de la descuidada formación inicial de profesores y licenciados en historia. Este aserto irroga un desarrollo mayor que excede los propósitos de este texto y que puede, empero, ser rastreado en los trabajos de Henríquez \& Canelo (2014) o el de Oteíza, Dalla Porta \& Garrido (2014).

Retomemos la hebra. Si se realiza una revisión, los propósitos curriculares más complejos de la disciplina, definidos en currículos y estándares, se 
presentan en el ámbito de la producción de textos escritos. En contraste, el currículo chileno de Historia y Ciencias Sociales (MINEDUC 2009; 2012; 2015) se focaliza en el aprendizaje de hechos y procesos ordenados cronológicamente y desconectados de la producción de textos. Aunque se propone el desarrollo de habilidades vinculadas a la lectura y escritura de textos históricos, estas quedan desvinculadas de los contenidos. En consecuencia, estas son divididas, por una parte, en habilidades de investigación histórica (análisis de evidencias) y, por la otra, en habilidades vinculadas tanto a la lectura de evidencias como a la producción escrita de una variedad de textos históricos (descriptivos, explicativos, etc.). Sumado a lo anterior, la evaluación nacional estandarizada, el Sistema de Medición de la Calidad en Educación (SIMCE) del sector Historia y Ciencias Sociales, se ha enfocado fundamentalmente en la medición de conceptos disciplinarios más que en habilidades argumentativas históricas. Los documentos oficiales no proporcionan evidencia concluyente que nos permita conocer de qué forma se desarrollan el pensamiento histórico y la escritura en las aulas chilenas. El estudio respecto de cobertura curricular en historia (MINEDUC, 2004) presenta pocos datos, limitándose a señalar porcentajes de cobertura de contenidos curriculares y no de habilidades. Incluiremos un ejemplo para ilustrar lo expuesto anteriormente.

Se presentan a continuación objetivos curriculares que cubren áreas del currículo donde no tenemos información respecto del modo como se evalúa o trabaja en las aulas (Cuadro 1). ¿Cómo sabemos que esos objetivos y contenidos son tratados en clases?, ¿cuáles son los resultados de los aprendizajes en las aulas chilenas y cómo se evalúa en ellas? Lo que llama la atención es que prácticamente todos los objetivos requieren la utilización del lenguaje oral o escrito para demostrar el logro del aprendizaje. ¿Cómo los evaluamos?, ¿mediante qué actividades de aula promovemos su desarrollo?

Como se puede apreciar en el Cuadro 1, en los fragmentos remarcados del cuadro anterior, existe una presencia no desdeñable de metas de aprendizaje marcadas por una marcada impronta escritural y que incluso explicita el uso perentorio de diversas fuentes documentales. Nuestra opinión es que, aun cuando el currículo estructure una distinción aparente entre habilidades heurísticas y de producción textual, puede constatarse longitudinalmente la presencia de prescripciones orientadas al desarrollo de habilidades de pensamiento histórico que remitirían hacia la adquisición de la gramática disciplinar misma, antes que de sus meros contenidos. En este sentido, el caso de $3^{\circ}$ Medio resulta particularmente decidor, toda vez que el análisis de procesos de la historia contemporánea de Chile reclamaría el uso integrado de herramientas de decodificación que permitieran al estudiante descifrar las operaciones escriturales que subyacen a las evidencias mismas que se utilizarán en la construcción de la interpretación propia. Por lo que, antes incluso de la comprensión textual del contenido documental de las evidencias, cabría preguntarse si los alumnos son capaces de dar cuenta de las operaciones historiográficas que inscriben la composición de la fuente, así como la estructuración de un relato con trama, orientación ideológica y propósito textual, entre otras cosas.

Se cuenta con algunas respuestas a las preguntas formuladas más arriba pero aún queda mucho por conocer de lo que sucede en las aulas. Quienes tienen un conocimiento más acabado son, sin duda, los y las profesores/as de aula, quienes reafirman, desde la experiencia, cuestiones que la literatura ha reportado desde otros contextos. Entre los hallazgos más frecuentes puede contarse la aseveración de que el desarrollo de prácticas de lectura, escritura y habla sería escaso en las aulas. Junto con ello, aparece la queja acerca de los currículos sobrecargados de contenidos factuales, la presión ministerial, de las familias e incluso de los propios establecimientos para que los profesores logren "pasar" por completo el currículo estatal.

Los reformadores curriculares aún están al debe con lo que nos han ofrecido a pesar de las reiteradas reformas curriculares: largos listados de contenidos y objetivos sin testeo previo, sin investigación consistente que los sustente y miles de páginas y recursos malgastados en su supuesta implementación. Lo anterior ha generado que las habilidades de lectura y escritura, que generalmente requieren de mucho tiempo, se dejen de lado para cubrir el listado de contenidos. Esto reporta escasas oportunidades para modelar y ejercitar el lenguaje académico en los estudiantes por medio de la producción de textos, como señalan estudios realizados en EE.UU. (Reisman, 2012; MonteSano, 2010; Fogo, 2014). La presencia de estas carencias, constatadas ya en el caso chileno, se exhibe también en el caso estadounidense, arrojando luces de alarma respecto del aprendizaje de la 
Cuadro 1. Objetivos de Historia y Ciencias Sociales.

Curso

$7^{\circ}$ Básico Unidad 1 (OA 01): Explicar el proceso de hominización, reconociendo las principales etapas de la evolución de la especie humana, la influencia de factores geográficos, su dispersión en el planeta y las distintas teorías del poblamiento americano.

Unidad 2 (OA 08): Analizar, apoyándose en fuentes, el canon cultural que se constituyó en la Antigüedad clásica, considerando la centralidad del ser humano y la influencia de esta cultura en diversos aspectos de las sociedades del presente (por ejemplo, escritura alfabética, filosofía, ciencias, historia, noción de sujeto de derecho, relaciones de género, ideal de belleza, deporte, teatro, poesía y artes, entre otros).

$8^{\circ}$ Básico Unidad 1 (OA 05): Argumentar por qué la llegada de los europeos a América implicó un enfrentamiento entre culturas, considerando aspectos como la profundidad de las diferencias culturales, la magnitud del escenario natural americano, y la desarticulación de la cosmovisión de las sociedades indígenas.

Unidad 3 (OA 16): Explicar la independencia de las colonias hispanoamericanas como un proceso continental, marcado por la crisis del sistema colonial, la apropiación de las ideas ilustradas y la opción por el modelo republicano, y analizar en este marco el proceso de Independencia de Chile.

$1^{\circ}$ Medio Unidad 1 (AE 05): Analizar, apoyándose en diversas fuentes de información, antecedentes, el impacto y la magnitud de la Segunda Guerra Mundial, incluyendo: > extensión planetaria y participación de millones de combatientes > uso de la ciencia y la tecnología para fines de destrucción masiva > gran mortandad de civiles > ideologías en pugna

Unidad 2 (AE 04): Analizar, integrando diversas fuentes de información, los principales procesos políticos de fines del siglo XX, considerando: > crisis del Estado de bienestar > auge del neoliberalismo y de las economías de libre mercado > caída del muro de Berlín y del comunismo en la URSS > fin de la Guerra Fría y nueva configuración mundial: predominio de Estados Unidos e importancia creciente de China, India y países árabes.

$2^{\circ}$ Medio Unidad 1 (AE 01): Explicar los cambios que se producen en la estructura social, jurídica y religiosa de los pueblos originarios de Chile con la llegada de los españoles y durante el período colonial, considerando: > contraste entre la destrucción y pervivencia de las culturas indígenas > disminución de la población originaria > procesos de sincretismo

Unidad 1 (AE 02): Analizar, contrastando distintas interpretaciones históricas, las relaciones entre españoles y mapuches a lo largo del periodo colonial, considerando:

> resistencia mapuche > guerra de Arauco y sus características > vida fronteriza en el Biobío: comercio e intercambio cultural > esclavitud en el siglo XVII > sistema de parlamentos en el siglo XVIII.

$3^{\circ}$ Medio Unidad 1 (AE 03): Analizar, a partir de diversas fuentes estadísticas y documentales, las transformaciones sociales hacia mediados del siglo XX (crecimiento demográfico y transformaciones urbanas; creciente escolarización; progresiva incorporación de las mujeres a la vida pública; nuevos medios de comunicación social y de entretención), y evaluar su incidencia en el proceso de democratización de la sociedad chilena.

Unidad 4 (AE 16): Analizar, apoyándose en diversas fuentes, los factores que inciden en el fin de la dictadura militar y los procesos que dan inicio a la transición a la democracia durante la década de 1980 (itinerario de la transición, formación de bloques políticos, plebiscito de 1988, negociación de las reformas constitucionales, elección presidencial de 1989).

$4^{\circ}$ Medio

Unidad 1 (AE 04): Comprender y analizar el funcionamiento del sistema judicial en Chile, considerando los principales rasgos del sistema procesal penal y civil, así como las responsabilidades penales y civiles, mediante casos reales y vigentes.

Unidad 3 (AE 15): Interpretar el concepto de región en el contexto de la Globalización y las influencias e impactos que este proceso produce en la realidad regional chilena, considerando los binomios: conectividad/ aislamiento, diversidad/homogeneidad, desarrollo económico/pobreza, flujos de capitales/ controles migratorios, entre otros, y la importancia de desarrollar una planificación territorial acorde a las nuevas dinámicas espaciales que configuran el espacio regional.

Fuente: Programas de Estudio de Historia y Ciencias Sociales (Ministerio de Educación, 2011a, 2011b, 2011c, 2011d, 2015a, 2015b). Citado en Henríquez y Mardones (2015). 
escritura histórica en estudiantes de aquel país. Por ejemplo, recientes resultados de lectura y escritura histórica evaluados por The National Assessment of Educational Progress (NAEP) durante el 2012 han mostrado que en la actualidad los estudiantes estadounidenses escriben historia peor que los de la década de 1990 (Shanahan \& Shanahan, 2008). Sin entrar en los detalles concernientes a las posibles causas, los investigadores han sostenido que la escasez de tiempo para desarrollar la lectura y la escritura, así como la falta de políticas curriculares de enfoque pedagógico que ofrezcan oportunidades a los profesores para el desarrollo de estas habilidades en las aulas, inciden en la generación de mejores condiciones para el aprendizaje (Moje 2008; MonteSano, De la Paz \& Felton, 2014).

Aunque el contexto de Estados Unidos sea muy distinto al chileno, algunos de los problemas del aprendizaje históricos brevemente indicados no son, como hemos ya afirmado, extraños para la realidad chilena. Investigaciones recientes identifican problemas similares en estudiantes chilenos de educación secundaria y terciaria a la hora de elaborar argumentos históricos a partir de evidencias, habilidad solicitada explícitamente, por ejemplo, en la Unidad 1 (OA 05), como se muestra en el Cuadro 1. Básicamente, estos problemas se vinculan con la dificultad para utilizar los argumentos de la evidencia como garantía necesaria de la elaboración de un nuevo argumento en un texto escrito (Henríquez \& Ruiz 2014; Henríquez \& Canelo, 2014; Oteiza, Dalla Porta \& Garrido, 2014).

En uno de esos estudios identificamos junto con Marcela Ruiz algunos rasgos discursivos presentes en textos escritos por estudiantes de $1^{\circ}$ Medio. La investigación tuvo una fase en la que implementamos una secuencia didáctica cuya meta fue la elaboración de una explicación histórica, como se solicita en las metas de aprendizaje que hemos expuesto para los niveles de $7^{\circ}$ básico, $8^{\circ}$ básico y $2^{\circ}$ Medio en el Cuadro 1. Estas secuencias fueron implementadas en el 2011 en 3 colegios de dependencia particular subvencionado de la comuna de Puente Alto de Santiago de Chile. Las secuencias estaban organizadas en un portafolio de 6 horas cronológicas, equivalente a 8 sesiones de clases de 45 minutos para cada curso participante. Su implementación contempló las siguientes etapas:

1. Contextualización.

2. Presentación del problema histórico.
3. Presentación y lectura de las evidencias (identificación, lectura en profundidad, conexión de las fuentes).

4. Organización de información de evidencias.

5. Selección de la información de las evidencias.

6. Jerarquización de la información de evidencias.

7. Planificación de escritura de la explicación.

8. Elaboración y entrega de explicación.

Los textos fueron elaborados utilizando fuentes primarias y secundarias. Una síntesis de los resultados puede consultarse en la Tabla 1. El hallazgo más significativo resultó ser que los estudiantes fueron escasamente competentes a la hora de elaborar explicaciones ante un problema histórico a partir de evidencias. Caracterizamos las respuestas en tres niveles por medio de la identificación de tres factores relevantes de la composición discursiva, a saber: la construcción de la agentividad (¿quién o qué realiza las acciones?), la conformación de la cadena causal (¿qué factor(es) explican la ocurrencia de un fenómeno?) y el establecimiento de la perspectiva histórica sobre la evidencia (¿qué grado de reconocimiento se posee acerca de la circunstancia en la que se inscribe la evidencia que se emplea?).

A continuación, reproducimos y analizamos algunas respuestas de estudiantes recopiladas a partir de la investigación empírica en los cursos de $8^{\circ}$ básico, $2^{\circ}$ Medio y $1^{\circ}$ Medio. Cada una de estas evidencias ha sido asociada a algunos de los niveles que pudimos proponer a la luz de los datos empíricos, los que categorizamos por medio de la siguiente forma:

\section{CASO 1: Crónica sin sentido histórico ( ${ }^{\circ}$ básico $)$}

Pregunta histórica: Durante los siglos XV y XVI se desarrollaron distintos viajes de exploración. En este contexto, Holanda e Inglaterra eran los países más desarrollados y con más tecnología para poder haber conquistado los continentes del mundo. No obstante España y Portugal, menos desarrolladas, lograron descubrir América. ¿Por qué estos reinos lograron formar la empresa de descubrimiento en América?

Respuesta: "En estos tiempos [TIEMPO DE LA CRÓNICA $]^{1}$ España y Portugal querían [VOLUNTAD-ANTECEDENTE] comercializar con Oriente porque sabían [ESTADO MENTAL] que podían ganar mucho dinero con el tema 
de las especias, telas, etc. Así [CONECTOR CONSECUTIVO] que ambos países decidieron [VOLUNTAD-CONSECUENTE] comercializar con ellos (Oriente)".

\section{CASO 2: Narración sin sentido histórico ( $2^{\circ}$ Medio)}

Pregunta histórica: América Latina a comienzos del siglo XIX era una colonia española. Cuando el rey de España fue tomado prisionero por los franceses, los criollos se mantuvieron leales al monarca, formando juntas de gobierno a la espera de que este fuera liberado.

Entonces, ¿Por qué los criollos que en un comienzo eran leales al rey, pasan a encabezar el proceso de independencia de las colonias americanas?

Respuesta: "Los criollos [GRUPO SOCIAL] estaban o eran leales al rey, aunque igual se quejaban [ESTADO EMOCIONAL-ANTECEDENTE] sobre el gobierno español y su administración [ENTIDAD INSTITUCIONAL] donde los criollos estaban excluidos [VALOR MOTIVACIONAL DE LA CONDICIÓN]. Ahora los criollos a través de las juntas de gobierno [ENTIDAD INSTITUCIONAL] llevaron proyectos de mejoramiento de las colonias [ENTIDAD INSTITUCIONAL] y eso los llevó a sentirse [ESTADOEMOCIONAL-CONSECUENTE] más apegados a Chile y a desear [VOLUNTAD] la independencia para que ellos pudieran incluirse en la organización del gobierno. Además cuando Fernando VII volvió al poder, los criollos sufrieron una represión, cual causó mucha molestia [ESTADO EMOCIONAL CONSECUENTE] en contra de la monarquía [ENTIDAD INSTITUCIONAL]".

\section{CASO 3: Narración con sentido histórico ( $1^{\circ}$ Medio)}

Pregunta histórica: Si la URSS y EE. UU. fueron aliados durante la Segunda Guerra Mundial, ¿por qué se convirtieron en potencias rivales tras finalizar este conflicto?

Respuesta: "Se convirtieron en rivales por su diferencia de ideas, esto comenzó en la conferencia de Yalta [ESTRUCTURA CONCEPTUAL-TEMPORALIDAD HISTÓRICA], ya que se quería separar a Alemania, como ambas partes querían dejar un gobierno distinto en Alemania empezó la pugna [ANTECEDENTE 1- HECHO
COMO CUASIMOTIVACIÓN]. Posterior a esto empezaron a atacarse mediante medios de comunicación de esta forma haciéndolo público, se puede citar la "doctrina Truman" [ESTRUCTURA CONCEPTUAL-TEMPORALIDAD HISTÓRICA] en la que Harry Truman deja en claro (de su punto de vista) que la vida norteamericana es mejor que en la URSS, también el discurso de Churchill que es contrarrestado por Stalin [ANTECEDENTE 1- PERSPECTIVA REFERENCIAL]".

En la Tabla 1 se expone de manera sintética el análisis realizado, a partir de los factores discursivos del texto (agentividad, construcción de la cadena causal y perspectiva de la evidencia) propuestos por Henríquez y Ruiz (2014), a cada uno de los desempeños estudiantiles asociados a los diversos niveles propuestos. Como se podrá apreciar, la variabilidad arrojada a la luz de la investigación empírica revela que los rendimientos más pobres están asociados a expresiones escriturales que integran solo de manera laxa los elementos fundamentales de la gramática académica de la disciplina histórica.

\section{Leer y escribir históricamente}

Las experiencias de aula que han implementado actividades y tareas de aula cuyo foco ha sido el desarrollo de habilidades de lectura y escritura histórica han señalado que estas estrategias constituyen una poderosa oportunidad no solo para el aprendizaje de la Historia, sino que también para el desarrollo de lectores y escritores más competentes en el marco de la disciplina histórica (Coffin, 2004; Moje, 2008; Monte-Sano, 2010; Reisman, 2012). ¿Qué han privilegiado estas propuestas de aula? El desarrollo de tareas de aula que promueven la lectura histórica, razonamiento histórico y escritura histórica. A continuación expandimos las implicancias de estas estrategias, ofreciendo nuevamente algunos ejemplos empíricos que ayudan a ilustrar su implementación.

a) Lectura histórica. El lector de este texto podrá interpelar a los autores del mismo acotando que la competencia lectora no debería ser algo enseñado propiamente tal por el área de Historia y Ciencias Sociales, sino más bien por el sector de Lenguaje y Comunicación. Sin embargo, los estudios acerca de prácticas de lectura han recalcado que las distintas comunidades disciplinares poseen prácticas de lectura y escritura dotadas de especificidades particulares. A raíz de lo anterior, 
Tabla 1. Síntesis de los factores discursivos de la explicación histórica.

\begin{tabular}{lll}
\hline & \multicolumn{1}{c}{ Agentividad } & Construcción de la cadena causal \\
\hline Crónica sin & Los actores son personajes y en- & Los factores históricos están vin- \\
sentido histórico & tidades con estados emocionales & culados por la sucesión temporal \\
(Nivel 1) & y mentales. & $\begin{array}{l}\text { como eje articulador. } \\
\text { Las marcas de temporalidad corres- } \\
\text { ponden al tiempo de la narración y } \\
\end{array}$ \\
& & con ausencia de cronología.
\end{tabular}

Narración sin Los actores son personajes y grupos sentido histórico sociales con estados emocionales intereses y propósitos. (Nivel 2) y mentales que poseen diferentes

Los factores históricos están vinculados por la sucesión temporal de los hechos a partir de un conflicto con una cronología imprecisa.

Los factores históricos están relacionados de forma unicausal.

Los factores históricos tienen un poder desencadenante equivalente, de modo que pueden funcionar indistintamente como antecedente y efecto.

Narración con Los actores son grupos sociales, sentido histórico entidades sociopolíticas y estruc(Nivel 3)
Los factores históricos están relacionados de forma multicausal y la cronología presenta una significación histórica limitada.

Los factores históricos son identificados y jerarquizados de acuerdo con su poder desencadenante, de esa manera los efectos históricos son los hechos y los antecedentes que los provocan corresponden a las motivaciones y las condiciones.
Las evidencias son sintetizadas sin alterar su aceptabilidad referencial. Se reconoce la procedencia de la evidencia, pero no se determina su importancia.

\begin{abstract}
Las evidencias son sintetizadas de acuerdo con el grado de pertinencia para responder al problema histórico.

Se reconoce la procedencia de la evidencia y se establecen vínculos intertextuales entre ellas; además se les atribuye un grado de valoración.
\end{abstract}

Fuente: Henríquez \& Ruiz (2014: 151).

algunas prácticas de lectura que resultan propias del trabajo específico de los historiadores son el trabajo con fuentes primarias y secundarias, el uso de estrategias de comprensión general o disciplinar, y las operaciones de identificación de la perspectiva del autor, comparación y corroboración de la información proveniente de diferentes documentos (Leinhardt \& Young, 1996; Wineburg, 1991a y b, 1998, 2001; Van Sledright, 2004; Paxton, 2002; Stahl \& Shanahan, 2004). Aunque muchas de estas estrategias, como releer, hacer esquemas y buscar el significado de los conceptos no conocidos, pueden ser propias de un buen lector, la particularidad de la lectura de los historiadores es la interpretación heurística de los textos. Es decir, poder evaluar el origen de los textos, reconocer al autor de estos, establecer su intención y circunscribir el contexto en que los documentos son elaborados, además de corroborar la fiabilidad de la información proporcionada en estos. De este modo, estas estrategias permiten ubicar al texto en un contexto temporal específico y establecer relaciones conceptuales con otros textos de la época (Wineburg, 1991). Esta habilidad de los historiadores integra para Young \& Leinhardt (1998) dos dimensiones del conocimiento disciplinar: el contenido del dominio y el conocimiento de los procesos retóricos de la disciplina. El acceso al conocimiento histórico se encuentra así mediado por el conocimiento que se tiene del lenguaje de la historia y su gramática. El aprendizaje del conocimiento disciplinar se lleva a cabo, como sostiene Halliday (2004), mediante textos orales y escritos elaborados con recursos lingüísticos particulares. El reconocimiento de estas dificultades ha propiciado algunos estudios en torno al proceso de adquisición de un lenguaje académico presente en la lectura y en la escritura de diferentes áreas disciplinares. Recientes estudios identifican que el discurso pedagógico de la Historia, así como el de otras disciplinas humanistas y sociales, comparte la peculiaridad de tener un lenguaje especializado con características particulares, como 
la presencia de nominalizaciones. Estas participan en la construcción abstracta de los conceptos y se traducen en un empaquetamiento o "cosificación" de los procesos y del tiempo. Asimismo propician la tendencia a la objetivación y la "existencialización" de los eventos, junto con el tratamiento impersonal de las relaciones causales que tienden a realizarse al interior de las cláusulas (Coffin, 2006; Martin, 2002; Martin \& Rose, 2008; Oteíza 2006, 2009, 2011 Oteíza \& Pinuer, 2012, 2013; Schllepegrell, 2004). Estas cualidades del discurso histórico permiten interpretar y explicar en el texto histórico las relaciones entre agentes, eventos y procesos, estableciendo causas y consecuencias entre ellos (Coffin, 2006).

b) Razonamiento histórico. En el contexto escolar se ha definido al razonamiento histórico como una actividad cognitiva que permite la organización de información sobre el pasado con el propósito de describir, comparar, o explicar fenómenos históricos (van Boxtel \& van Drie, 2008). Para llevar a cabo dicho propósito resulta necesario realizar algunas tareas de aula, como realizar preguntas históricas, contextualizar la evidencia, hacer uso de conceptos sustantivos y metaconceptos de la disciplina histórica, además de sustentar sus afirmaciones en argumentos basados en la evidencia de las fuentes que dan información acerca del pasado. La calidad del razonamiento histórico de los estudiantes está influenciada por la naturaleza de la tarea, el tópico o tema, así como por el material histórico entregado (van Boxtel \& van Drie, 2008). Por ello, un número relevante de las dificultades que experimentan los alumnos para comprender las preguntas históricas, los textos y las evidencias está vinculado con los problemas que estos padecen a la hora de "desempaquetar" o retraducir el conocimiento "empaquetado" por la acción del lenguaje académico y algunos de sus fenómenos propios, como las nominalizaciones.

c) Escritura histórica. Estudios provenientes de la Lingüística Sistémico-Funcional (LSF) han aportado categorías para describir el lenguaje académico que utiliza la disciplina histórica en la escritura. Tal caracterización permite focalizar las tareas de aula para resolver las dificultades del aprendizaje histórico dadas por la distancia existente entre el saber de la experiencia cotidiana y el conocimiento académico de la disciplina (Halliday \& Martin, 1993). Debido a que el lenguaje de las disciplinas científicas entraña una visión del mundo diferente respecto de la experiencia cotidiana, muchas de las problemáticas del aprendizaje que experimenta el neófito pueden enlazarse a dificultades de acceso al lenguaje abstracto construido sobre relaciones conceptuales al interior de la disciplina (Halliday \& Martin, 1993; Halliday, 2004; Martin, 2011). La teoría de los géneros, desarrollada por la Escuela de Sidney, proporciona un conjunto de herramientas que permiten caracterizar el uso y los significados presentes en los textos. De esta forma, un género es entendido como un proceso social orientado a fines que presentan cierta estructura organizativa (Coffin, 2006; Martin \& Rose, 2008; Martin, 2009). En el caso de la disciplina en estudio, los géneros históricos escolares han sido caracterizados (Coffin, 2006; Martin \& Rose, 2008) a partir de diferentes registros que se intersectan en las interacciones del ecosistema de la escuela, a saber: textos producidos por estudiantes de educación básica y secundaria (Coffin, 2006), libros de textos de Historia (Oteiza, 2009; 2011; Altamirano, Godoy, Manghi, \& Soto 2014) e interacciones de clase entre los diversos sujetos participantes (Manghi 2013; Manghi, Badillo \& Villacura, 2014). La producción de textos históricos tiene particularidades que permiten agruparlos en a) géneros narrativos, en los que prima el tiempo del campo y, b) en géneros argumentativos, en los que prima el tiempo del texto (Coffin, 2006). Las principales características del primero consisten en relacionar eventos concretos y particulares, inscritos en una temporalidad lineal. En la enseñanza de la disciplina histórica, el género narrativo es uno de los más utilizados, porque contribuye a resumir eventos históricos, insertándolos en una secuencia temporal lineal (Martin \& Rose, 2008; Coffin, 2006). En su contraste, los géneros argumentativos se diferencian de los narrativos porque incorporan relaciones causales en la secuencia temporal principal, es decir, en vez de unir solo temporalmente los hechos de manera consecutiva (uno tras otro), estos adquieren un papel agentivo que provoca o explica eventos subsecuentes (Coffin, 2006).

En nuestra opinión, es a partir de la caracterización y la implementación racional de los "géneros históricos" que será posible adecuar las secuencias didácticas, las prácticas de lectura y las tareas de escritura a los propósitos curriculares y didácticos en cada uno de los contextos de implementación de la investigación.

En una línea similar, un estudio realizado con estudiantes de $8^{\circ}$ básico de un colegio particular 
subvencionado de la comuna de La Florida de Santiago de Chile, mostró que la calidad de las argumentaciones históricas de los alumnos está directamente relacionada con la capacidad de comprender la información de la evidencia y con la utilización de recursos léxico-gramaticales para su incorporación en un texto escrito (Henríquez, Carmona \& Quinteros, en evaluación). Junto con los profesores de aula Andrés Carmona y Alen Quinteros se establecieron cuatro etapas en el desarrollo del género del "recuento histórico" con sus relativas fases de progreso. A partir de la evidencia secundaria (Coffin, 2006; Martin \& Rose, 2008) hemos recogido las siguientes etapas:

\section{1. inicio \\ 2. contextualización \\ 3. reformulación \\ 4. cierre}

En esta investigación se utilizó un diseño cualitativo con estrategias de análisis del discurso basadas en la perspectiva de la Lingüística SistémicoFuncional (LSF). Este enfoque utiliza la noción de "género" entendiéndolo como las opciones recurrentes de elecciones semánticas que se desarrollan en etapas y apuntan a la realización de un propósito social compartido culturalmente (Martin \& Rose 2008). Como nuestro propósito de investigación se focalizó en caracterizar la elaboración del sentido histórico en estudiantes de $8^{\circ}$ básico, en un contexto escolar y curricular situado, se trabajó con el AE 01 de la Unidad 2 del currículo, el que indica: " [D] imensionar geográficamente la expansión de la influencia europea hacia otros continentes a través de procesos de exploración y conquista durante la época Moderna".

Las fases de progreso de cada una de las etapas transitan desde menores a mayores niveles de abstracción, así como entre un uso más escaso y uno más denso o frecuente de la evidencia proporcionada a los alumnos. La caracterización de 4 etapas se estableció a partir de la identificación de los rasgos léxico-gramaticales más preponderantes en las respuestas de los estudiantes. Solo a partir de esto se definieron las fases de progreso antes señaladas (Cuadro 2). El tiempo de implementación fue de 10 horas pedagógicas y consistió fundamentalmente en clases expositivas y en el trabajo con un portafolio de 3 mapas correspondientes a diferentes épocas históricas que describían la expansión europea. La producción del texto final individual tuvo la finalidad de responder a la siguiente pregunta histórica:

¿Cómo la evolución del conocimiento geográfico representado en estos documentos refleja la expansión del mundo europeo? (Henríquez, Carmona \& Quinteros, en evaluación).

Presentamos a continuación algunos ejemplos en los que se pueden observar las etapas y fases de realización en un escrito que responde a la pregunta histórica planteada a los alumnos. A continuación se indica la etapa, la fase y los ejemplos:

Cuadro 2. Fases y etapas del género "recuento histórico".

\begin{tabular}{|c|c|c|c|c|}
\hline $\begin{array}{l}\text { Fases } \\
\text { Etapas }\end{array}$ & $\begin{array}{c}\text { Menor uso de evidencia } \\
\text { Menos abstracción }\end{array}$ & & & $\begin{array}{c}\text { Mayor uso de evidencia } \\
\text { Mayor abstracción }\end{array}$ \\
\hline $\begin{array}{l}\text { Inicio } \\
\text { Adhesión al propósito }\end{array}$ & Ausencia de propósito & \multicolumn{2}{|l|}{ Inversión del propósito } & $\begin{array}{l}\text { Reformulación } \\
\text { de la pregunta }\end{array}$ \\
\hline $\begin{array}{l}\text { Contexto } \\
\text { Inclusión de tiempo y } \\
\text { espacio }\end{array}$ & \multicolumn{2}{|l|}{ Ausencia de evidencia } & \multicolumn{2}{|c|}{ Inclusión de referencias temporales y espaciales } \\
\hline $\begin{array}{l}\text { Reformulación } \\
\text { Inclusión de la evidencia } \\
\text { y perspectiva sobre ella }\end{array}$ & Ausencia de evidencia & Crónica factual & $\begin{array}{l}\text { Crónica orientada } \\
\text { a consecuencias }\end{array}$ & $\begin{array}{l}\text { Narración basada en } \\
\text { evidencia }\end{array}$ \\
\hline $\begin{array}{l}\text { Cierre } \\
\text { Uso de evidencias }\end{array}$ & $\begin{array}{l}\text { Orientación por avance } \\
\text { del tiempo }\end{array}$ & \multicolumn{2}{|c|}{ Orientación por interpretación } & $\begin{array}{l}\text { Síntesis con narración y } \\
\text { explicación }\end{array}$ \\
\hline
\end{tabular}




\section{A. Etapa Inicio. Fase Inicio de inversión del propósito.}

Ejemplo 1.

"La evolución de conocimiento geográfico permite la expansión del mundo".

En este ejemplo, el estudiante invierte la relación entre la causa (expansión geográfica) y la consecuencia (el conocimiento geográfico) que se proponen en el problema, ya que comprende que es la expansión del mundo conocido la que refleja la evolución del conocimiento geográfico ( $c f$. pregunta histórica propuesta).

\section{B. Etapa contexto. Fase de inclusión de tiempo o} espacio. Se incluye la referencia temporal (siglos) y el lugar donde fueron elaborados los mapas

Ejemplo 2.

"Estas fuentes fueron hechas entre el siglo XI y el siglo XIII, la primera fuente fue hecha por ingleses en el siglo XI, la segunda fuente fue hecha por Portugal en el siglo XVI y la tercera fuente fue hecha en francia [sic] el siglo 18".

En este ejemplo, el alumno logra el nivel máximo de la etapa de contexto, porque incluye en su respuesta la referencia temporal ("entre el siglo XI y el siglo XIII") y espacial ("fue hecha por Portugal") en la que las evidencias proporcionadas fueron elaboradas.

\section{Etapa Reformulación. Fase de crónica factual.}

Ejemplo 3.

"Bueno, en un principio en la edad media el hombre europeo era teocéntrico y no ocupaban la razón tanto en relación al renacimiento y Humanismo aún así ellos conocían Europa, Asia y África en una parte, aunque ellos pensaban que esa parte de África era Asia, el mapa y sus conocimientos teocéntricos se demuestran porque al centro de este mapa se encontraba tierra Santa (Jerusalén)".

La reformulación se realiza a partir de la construcción cronológica de la temporalidad con expresiones como "principio" y "edad media" junto con verbos en pasado y presente ("era", "ocupaban", "conocían", "pensaban", "encontraba"). Además se constata una expresión congruente de la causalidad formulada por medio de la conjunción "porque", la que le confiere el carácter de crónica a los textos de los estudiantes, toda vez que les permite enfatizar no un tiempo del texto (agentivo o explicativo), sino el de una narración lineal de los hechos.

\section{Etapa Cierre. Fase de orientación por avance del tiempo.}

Ejemplo 4.

"El conocimiento geográfico refleja la expansión del mundo europeo, ya que cada mapa es más avanzado que el anterior y la mayoría de las tierras representadas estaban bajo el control de algún país europeo, porque no SOLO vinieron a descubrir, sino a conquistar, hacerse más poderosos, y conocer más".

Las conclusiones se realizan a partir de la construcción congruente de la causalidad por medio una conjunción ("ya que"), que genera el efecto de avance y la demarcación del tiempo. En este caso, puede constatarse además un uso atributivo de ciertas cualidades históricas (progreso) a la sucesión temporal mediante la coordinación de las palabras "avanzado" y "anterior". Como se ha expuesto ya, la linealidad temporal de los hechos como eje rector del texto resulta ser un recurso propio de los géneros narrativos arraigados en la crónica, de los que el "recuento histórico" (Coffin, 2006) es uno más.

En grandes rasgos, el principal logro de esta investigación exploratoria fue identificar cuatro etapas (inicio, contextualización, reformulación y cierre) y diferentes fases de progreso del género "recuento histórico". La gradación de estas etapas se estipuló, como ya se ha mostrado, a partir de la variabilidad en los niveles de abstracción, así como la frecuencia y extensión del uso documental realizado por los alumnos. No obstante lo anterior, el estudio previo referenciado ha contribuido asimismo a entregar estrategias de evaluación que permitan someter a interrogación el modo en que se puede cotejar el avance de los alumnos en cuanto a estas habilidades ministerialmente prescritas que exigen la elaboración de desempeños escriturales. De este modo, a partir de la estipulación de estas etapas y fases se ha podido construir un marco de referencia para clasificar los desempeños del alumnado, pero también para asistir de manera más estable a las necesidades de retroalimentación y mejoría que la actividad de aula reclama.

De manera complementaria, es posible señalar además que las características anteriormente mentadas traspasan la educación secundaria y se manifiestan también en el nivel terciario. Los estudios acerca de alfabetización histórica en estudiantes universitarios de Historia (Henríquez \& Canelo, 2014; Oteiza, Dalla Porta, Garrido, 2014) han mostrado que la construcción de explicaciones históricas de estos alumnos se presentan con mayor o menor peso 
argumentativo y con mayor o menor abstracción con directa dependencia de los tipos de recursos léxico-gramaticales utilizados por estos a la hora de incluir la perspectiva de las evidencias en la escritura y elaborar así la significación histórica de los textos. En todos los estudios mencionados se ha concluido que la escasez de actividades de aula que promuevan la lectura y escritura disciplinar posee una incidencia en la manifestación de problemas de aprendizaje de los niveles más complejos de cada una de las disciplinas académicas.

\section{Conclusiones}

Leer y escribir históricamente es una de las actividades pedagógicas que más contribuye al desarrollo de los propósitos curriculares y del pensamiento histórico. Sin embargo, los métodos de alfabetización histórica que la promueven (prácticas de lectura, razonamiento y escritura histórica) no han recibido suficiente atención ni en los currículos ni en las prácticas de aula. Esto por diferentes motivos: el currículo no proporciona, a nuestro juicio, las herramientas ni los mecanismos suficientes para elaborar evidencia concerniente a la calidad cabal de su implementación. Al respecto, las evaluaciones estandarizadas también han resultado limitadas a la hora de medir la adquisición de ciertas habilidades que exigen, para convertirse en evaluables, la elaboración de productos de aprendizaje como la escritura.

Por otro lado, a partir de lo revisado en este texto, nos resulta plausible afirmar que el currículo de Historia y Ciencias Sociales evidencia una arquitectura que provoca un efecto disociador de los procesos heurísticos que componen la comunicación humana (decodificación, elaboración de mensajes, interpretación, etc.). En este sentido, el exceso de contenidos y la falta de tiempo y herramientas para la implementación de habilidades de alfabetización histórica no hacen sino contribuir a la acentuación de una tendencia orientada a la pauperización del repositorio de competencias disciplinares que puede proponer la disciplina historiográfica.

Respecto de esto, la evidencia ofrece material suficiente para evaluar como plausible una configuración de la enseñanza que no dé relieve solamente a la transmisión de relatos históricos, sino también, y de manera fundamental, a las destrezas de alfabetización que hacen posible la operatoria con evidencias, puntos de vista y géneros que configuran la morfología de los productos escritos en el campo de la historiografía. No obstante lo anterior, un aspecto al que, en nuestro criterio, urge poner atención de manera perentoria se vincula fundamentalmente al establecimiento de canales, formas o instrumentos de evaluación que permitan sondear las áreas oscuras de un currículo que, si bien no desconoce el desarrollo de habilidades conducentes a la adquisición del pensamiento histórico, sí lo disocia del curso de aprendizaje de los contenidos y lo mantiene como una auténtica "caja negra" a la que solo pueden acceder, hoy por hoy, los docentes de aula que tienen la oportunidad de cotejar en vivo el desarrollo de competencias escriturales.

La caracterización de los recursos históricodiscursivos proporciona evidencia consistente respecto de la adquisición del pensamiento histórico por medio de sus huellas en el lenguaje académico disciplinar. Además, nos permite clarificar las oportunidades de aprendizaje y las prácticas de enseñanza que favorecen su desarrollo (formulación de preguntas históricas, prácticas de lectura de evidencia, prácticas de escritura histórica, retroalimentación). Adicionalmente, una mayor cuota de conciencia respecto de los recursos escriturales movilizados en el desarrollo de la disciplina arroja también una luz importante a la hora de ofrecer tipologías de fenómenos conducentes a colaborar, desde el punto de vista del estudioso o el docente, en la evaluación y análisis de la evidencia escrita elaborada por los alumnos. Consideramos que la incorporación de técnicas y métodos de análisis provenientes de la Lingüística SistémicoFuncional colabora en este derrotero, debido a que, como expusimos en los ejemplos vinculados a los tres niveles de desarrollo narrativo en historiografía, nos permite catalogar la función que las partículas y huellas textuales realizan en la escritura (agencia, relaciones de temporalidad, tipos de causalidad, etc.), ensanchando con ello una puerta de entrada a la comprensión del modo en que los estudiantes aprenden a trabajar en la disciplina histórica.

Según nuestra apreciación, hay una segunda disociación aparente que no se debe seguir repitiendo si es que se busca implementar mejorías en el aprendizaje disciplinar: la ruptura entre las habilidades de decodificación y utilización de los sistemas disciplinares en el área de Lenguaje y Comunicación y la de Historia y Ciencias Sociales. A juicio nuestro, esta fragmentación corre el riesgo de ser ilusoria toda vez que no se exprese con 
claridad que los lenguajes disciplinares, antes que ser repositorios conceptuales y gramaticales naturales, son producto de un aprendizaje que, aun teniendo como base la adquisición de habilidades básicas de la comunicación humana, comporta especificidades no desdeñables toda vez que se las enfoca desde el campo mismo de la disciplina histórica. Una de las hipótesis que hemos tratado de circunscribir por medio de este estado del arte se vincula con la idea de que el aprendizaje del lenguaje disciplinar, su elucidación y destreza de uso están emparejados con el empleo extensivo, permanente y mediado de fuentes históricas e instancias de aprendizaje y ejercitación de la escritura en el aula.

$\mathrm{El}$ argumento al que nos plegamos luego de este balance estriba en que la alfabetización histórica está lejos de ser un proceso desordenado y azaroso, ya que ella sigue fases que se refrendan por medio de la experiencia de análisis de las evidencias elaboradas por los propios estudiantes. La tendencia que hemos sondeado es que a medida que avanzan los niveles de abstracción y lenguaje disciplinar específico de la historia aparecen también la coocurrencia de un uso más extensivo, constante y profundo de la evidencia histórica, así como también un uso racionalizado y consciente de las posibilidades de los géneros históricos partícipes en la disciplina.

Por último, consideramos preciso recalcar que aun cuando la mayor parte de la evidencia relativa al estudio del pensamiento histórico ha sido desarrollada en contexto anglosajón, hay elementos que sí revisten informaciones que, sin extrapolarse, llaman a tener notas de atención aplicables para el caso nacional. En particular, la experiencia evaluativa de la NAEP, en donde se consignó el drástico deterioro de las competencias escriturales en el contexto norteamericano, no hace sino poner un foco de alerta y preocupación respecto de la elaboración de instrumentos y políticas de nivel sistémico que consideren la posibilidad de evaluar de manera más fina e intensiva los rasgos escriturales que han circunscrito esta llamada "caja negra" del currículo. Es innegable, según pensamos, el reconocimiento de ciertos umbrales de agencia a nivel docente respecto de qué se puede hacer para trabajar o favorecer instancias de aprendizaje destinados a desarrollar estas desatendidas habilidades, no obstante, recalcamos que se trata fundamentalmente de interrogarse acerca del diseño mismo del currículo y respecto de aquellas condiciones arquitectónicas que exceden el espacio de acción docente. Al igual que en el caso de los estudiantes que muestran mejores logros a nivel de las competencias históricas, la respuesta parecer ser semejante en el nivel macroestructural: urge la necesidad de implementar políticas y diseños empíricamente fundados en la evidencia y en la capacidad de poder medirlos, con el fin de monitorearlos. La respuesta es, a fin de cuentas, una de las preguntas sustanciales a indagar: ¿cómo evaluar el pensamiento histórico?

\section{Referencias Citadas}

Altamirano, P.; Godoy, G.; Manghi, D.; \& Soto, G. 2014 Analizando los textos de Historia, Geografía y Ciencias Sociales: La configuración multimodal de los pueblos originarios. Estudios pedagógicos (Valdivia), 40(1), 263-280. Coffin, $C$.

2004 Learning to write history: The role of causality. Written Communication, 21(3), 261-289.

Coffin, C.

2006 Historical discourse: The language of time cause and evaluation. London: Continuum.

Fogo, B.

2014 Core Practices for Teaching History: The Results of a Delphi Panel Survey. Theory \& Research in Social Education, 42, 2, 151-196.

Halliday, M. \& Martin, J.

1993 Writing Science: Literacy and Discursive Power. London: Falmer (Critical Perspectives on Literacy and Education). Halliday, Michael

2004 The Language of Science. London: Continuum (Collected Works of M. A. K. Halliday, Vol. 5).
Henríquez, R. \& Mardones, R.

2015 "Educación y ciudadanía". En Sánchez, I. (edit.). Ideas en Educación. Reflexiones propuestas. Santiago: Ediciones UC, pp. 572-600.

Henríquez, R. \& Canelo, V.

2014 Géneros históricos y construcción de la significación histórica en estudiantes de Licenciatura en Historia. Onomazein. Número Especial IX ALSFAL, 138-160.

Henríquez, R. \& Ruiz, M.

2014 Chilean students learn to think historically: Construction of historical causation through the use of evidence in writing. Linguistics and Education, 25, 1, 145-157.

Henríquez, R.; Carmona, A.; Quinteros, A. (en revisión) Escribir historia desde las evidencias. Género "recuento histórico" en estudiantes de $8^{\circ}$ de Educación Básica.

Henríquez, R.

2008 "El aprendizaje de la explicación y la comprensión histórica: la construcción narrativa del pasado y la conciencia histórica de los alumnos inmigrantes en Cataluña", Tesis de doctorado. Universidad Autónoma de Barcelona. 
Lee, $\mathrm{P}$.

2002 "Walking backwards into tomorrow". Historical consciousness and understanding history Paper given at Annual Meeting of American Educational Research Association, New Orleans.

Lee, $\mathrm{P}$.

2005 Putting principles into practice understanding history. En Donovan, M. \& Bransford, J. (Eds.). How Students Learn: History in the Classroom. Washington, D.F.: National Academies Press.

Manghi, D.

2013 Géneros en la enseñanza escolar: Configuraciones de significado en clases de historia y biología desde una perspectiva multimodal. Revista signos, 46(82), 236-257.

Manghi, H.D.; Badillo, V.C.; \& Villacura, A.P.

2014 Alfabetización semiótica en clases de Historia Estrategias de mediación desde un enfoque multimodal. Perfiles educativos, 36(146), 63-79.

Martin, J. \& White, P.

2005 The Language of Evaluation: Appraisal in English. Londres: Palgrave MacMillan.

Martin, J.

2009 Genre and language learning: A social semiotic perspective. Linguistics and Education, 20, 1, 10-21.

Martin, J.

2011 Prólogo. En Teresa Oteiza Silva, \& Derrin Pinto (Eds.), En (re)construcción: Discurso, identidad y nación en los manuales escolares de historia y de ciencias sociales (pp. 11-17). Santiago, (Chile): Editorial Cuarto Propio.

Martin, J.

2002 Writing History: Construing Time and Value in Discourses of the Past. En: Mary Schleppegrell \& Cecilia Colombi. Eds. Developing Advanced Literacy in First and Second Languages: Meaning with Power. Mahwah, NJ: Lawrence Erlbaum Associates.

Martin, J. \& Rose, D.

2008 Genre Relations: mapping culture. London: Equinox.

Ministerio de Educación, Chile MINEDUC

2009 Mapas de progreso del aprendizaje. Sector Historia, Geografía y Ciencias Sociales. Mapa de progreso de sociedad en perspectiva histórica. Retrieved from: http://www.mineduc. cl/index5_int.php?id_portal=47\&id_contenido $=17116 \&$ id seccion $=3264 \& c=362$

Ministerio de Educación, Chile, MINEDUC

2012 Bases Curriculares de la Educación Básica. Historia y Geografía y Ciencias Sociales. Santiago Gobierno de Chile. Ministerio de Educación. http://www.mineduc.cl/ index5_int.php?id_portal=47\&id_contenido $=17116 \&$ id seccion $=3264 \& \mathrm{c}=1$.

Ministerio de Educación, Chile, MINEDUC

2015 Programa de Estudio. Historia, Geografía y Ciencias Sociales. Tercer año medio. Retrieved from: www.curriculumenlineamineduc.c1/605/articles-30013_recurso_30_2. pdf

Ministerio de Educación, Chile, MINEDUC

2004 Cobertura curricular en segundo ciclo básico y enseñanza media subsectores: Estudio y Comprensión de la Sociedad- Historia y Ciencias Sociales. Retrieved from:http://curriculumenlinea.mineduc.cl/sphider/search. php?query $=\&$ t_busca $=1 \&$ results $=\&$ search $=1 \&$ dis $=0 \& \mathrm{ca}$ tegory $=6777$
Moje, E.B.

2008 Foregrounding the disciplines in secondary literacy teaching and learning: A call for change. Journal of Adolescent and Adult Literacy, 52(2), 96-107.

Monte-Sano, C.

2010 Disciplinary literacy in history: An exploration of the historical nature of adolescents' writing. Journal of the Learning Sciences, 19(4), 539-568.

Monte-Sano, C.; De La Paz, S.; Felton, M.

2014 Implementing a disciplinary-literacy curriculum for US history: learning from expert middle school teachers in diverse classrooms. Journal of Curriculum Studies, 46(4), 540-575.

Monte-Sano, C.; De la Paz, S.; Felton, M.

2014 Reading, thinking, and writing about history. NY. Teachers College Press.

Oteiza, R. y Pinuer, C.

2013 "El factor linear en la construcción del significado valorativo en el discurso". RILCE, Revista de Filología Hispánica, 29.2, pp. 443-472.

Oteiza Teresa y Claudio Pinuer

2012 Prosodia valorativa: construcción de eventos y procesos en el discurso de la historia. Discurso \& Sociedad, 6(2), pp. 418-446.

Oteiza, T.; Garrido, M.; Dalla Porta, C.

2014 La evidencialidad en la construcción de la significación histórica por estudiantes de Licenciatura de Historia. Onomázein, 57-80.

Oteiza, Teresa

2006 El discurso pedagógico de la historia. Un análisis lingüístico sobre la construcción ideológica de la historia de Chile (1970-2001). Santiago, Chile: Frasis editores.

Oteiza, Teresa

2009 Solidaridad ideológica en el discurso de la historia: tensión entre orientaciones monoglósicas y heteroglósicas. Revista Signos. Estudios de Lingüística 42 (70), pp. 219-244.

Oteiza, Teresa

2011 Representación de las memorias del pasado: intersubjetividad en el discurso pedagógico de la historia. En Teresa Oteiza y Derrin Pinto. En (re)construcción: Discurso, identidad y nación en los manuales escolares de historia y de ciencias sociales. Santiago (Chile): Editorial Cuarto Propio, pp. 129-172.

Paxton, R.J.

2002 The Influence of Author Visibility on High School Students Solving a Historical Problem. Cognition and Instruction, 20(2), 197-248.

Reisman, A.

2012 Reading Like a Historian: A Document-Based History Curriculum Intervention in Urban High Schools. Cognition and Instruction, 30(1), 86-112.

Rüsen, J.

1992). "El desarrollo de la competencia narrativa en el aprendizaje histórico. Una hipótesis ontogenética relativa a la conciencia moral". Propuesta Educativa No 7 , FLACSO, Buenos Aires.

Schleppegrell Mary

2004 The Language of Schooling. A Functional Linguistics Perspective. London: Routledge.

Schleppegrell, M.J.; Achugar, M.; Oteíza, T.

2004 The Grammar of History: Enhancing Content-Based Instruction Through a Functional Focus on Language. TESOL quarterly, 38(1), 67-93. 
Schleppegrell, M.J.; Greer, S.; Taylor, S.

2008 Literacy in history: Language and meaning. Australian Journal of Language and Literacy. 3 (2), 174-187.

Schleppegrell, M.; de Oliveira, L.C.

2006 An integrated language and content approach for history teachers. Journal of English for Academic Purposes, 5(4), 254-268.

Shanahan, T.; Shanahan, C.

2008 Teaching disciplinary literacy to adolescents: Rethinking content area literacy. Harvard Educational Review, 78(1), 40-61.

Snow, C.; Uccelli, P.

2008 A Research Agenda for Educational Linguistics. En Bernard Spolsky \& Francis Hult (Eds.) The handbook of educational linguistics. Singapur: Blackwell.

Stahl, S.; Shanahan, C.

2004 Learning to think like a Historian. Disciplinary Knowledge through Critical Analysis of Multiple Documents. In T. L. Jetton \& J. A. Dole (Eds.), Adolescent literacy research and practice (pp. 94-115). New York: Guilford Press.

Van Boxtel, C.; Van Drie, J.

2008 Historical reasoning: Towards a framework for analyzing students' reasoning about the past. Educational Psychology Review, 20(2), 87-110.

VanSledright, B.

2004 "What does it mean to think historically... and how do you teach it". Social Education, 68(3), 230-233.
Wineburg, S.

1991a Historical problem solving: A study of the cognitive processes used in the evaluation of documentary and pictorial evidence. Journal of Educational Psychology Journal of Educational Psychology, 83(1), 73-87.

Wineburg, S.

1991b On the Reading of Historical Texts: Notes on the Breach Between School and Academy. American Educational Research Journal American Educational Research Journal, 28(3), 495-519.

Wineburg, S.

1998 Reading Abraham Lincoln: An Expert/Expert Study in the Interpretation of Historical Texts. Cognitive Science, 22(3), 319-346.

Wineburg, S.

2001 Historical thinking and other unnatural acts: charting the future of teaching the past. Philadelphia: Temple University Press.

Wineburg, S.; Martin, D.; Monte-Sano, C.

2011 Reading like a historian: Teaching literacy in middle and high school classrooms. New York: Teachers College Press.

Young, K. M., \& Leinhardt, G.

1998 Writing from primary documents: A way of knowing in history. Written Communication, 15 (1), 25-68.

\section{Nota}

1 Los conceptos entre corchetes [ ] son marcas que se utilizaron para caracterizar los rasgos histórico-discursivos de las respuestas. 
